\title{
PIPE-chipSAD: A Pipeline for the Analysis of High Density Arrays of Bacterial Transcriptomes
}

\section{OPEN ACCESS}

Edited by:

Francesco Nicassio,

Istituto Italiano di Tecnologia (IIT), Italy

Reviewed by:

Astrid Desiree Haase,

National Institute of Diabetes and Digestive and Kidney Diseases (NIH),

Eleonora Lusito, Istitute of Molecular Oncology

Foundation (IFOM), Italy

*Correspondence:

Silvia Bottin

silvia.bottini@unice.fr

Alessandro Muzzi

alessandro.x.muzzi@gsk.com

${ }^{\dagger}$ Present Address:

Silvia Bottini

Centre Méditerranéen de Médecine Moléculaire, Institut National de la Santé et de la Recherche Médicale

U1065, Nice, France; Luca Fagnocchi,

Department of Epigenetics,

Fondazione Istituto Nazionale di Genetica Molecolare "Romeo ed

Enrica Invernizzi", Milan, Italy

${ }^{\ddagger}$ These authors have contributed equally to this work.

Specialty section: This article was submitted to Ribonucleoprotein Networks, a section of the journal Frontiers in Molecular Biosciences

Received: 05 September 2016 Accepted: 30 November 2016 Published: 20 December 2016

Citation:

Bottini S, Del Tordello E, Fagnocchi L,

Donati C and Muzzi A (2016)

PIPE-chipSAD: A Pipeline for the

Analysis of High Density Arrays of Bacterial Transcriptomes.

Front. Mol. Biosci. 3:82. doi: 10.3389/fmolb.2016.00082

\author{
Silvia Bottini ${ }^{1 *+}$, Elena Del Tordello ${ }^{1 \neq}$, Luca Fagnocchi ${ }^{1+\neq}$, Claudio Donati ${ }^{2}$ and \\ Alessandro Muzzi ${ }^{1 *}$ \\ ${ }^{1}$ GSK Vaccines Srl, Siena, Italy, ${ }^{2}$ Computational Biology Unit, Research and Innovation Centre, Fondazione Edmund Mach, \\ San Michele all'Adige, Italy
}

PIPE-chipSAD is a pipeline for bacterial transcriptome studies based on high-density microarray experiments. The main algorithm chipSAD, integrates the analysis of the hybridization signal with the genomic position of probes and identifies portions of the genome transcribing for mRNAs. The pipeline includes a procedure, align-chipSAD, to build a multiple alignment of transcripts originating in the same locus in multiple experiments and provides a method to compare mRNA expression across different conditions. Finally, the pipeline includes anno-chipSAD a method to annotate the detected transcripts in comparison to the genome annotation. Overall, our pipeline allows transcriptional profile analysis of both coding and non-coding portions of the chromosome in a single framework. Importantly, due to its versatile characteristics, it will be of wide applicability to analyse, not only microarray signals, but also data from other high throughput technologies such as RNA-sequencing. The current PIPE-chipSAD implementation is written in Python programming language and is freely available at https://github.com/silviamicroarray/chipSAD.

Keywords: high density arrays, tiling arrays, microarrays, transcriptomes, code:python

\section{INTRODUCTION}

The rapid development of new and high-throughput technologies to conduct genome-wide studies has dramatically increased the ability to discover new non-coding regulatory RNAs. Among the several transcriptome-profiling methods (Zhang et al., 2014), high-density DNA tiling microarrays (Selinger et al., 2000) have been successfully applied to a variety of transcriptome studies (Aparicio et al., 2004; Bertone et al., 2004; Weber et al., 2005; Crawford et al., 2006; Liu, 2007; Heidenblad et al., 2008).

The analysis and interpretation of high density microarray data is based on a precise definition of discrete transcriptional units, thus requiring a specific algorithm to identify them. In particular, the main challenge is to segment the hybridization signal along the genomic coordinate to accurately obtain transcripts boundaries, especially when also non-coding regions are probed. Different statistical algorithms have been developed to process high-density array data and to obtain such segmentation. The widest exploited method was introduced by Kampa et al. (Kampa et al., 2004) and was successively implemented in the Tiling Array Software (TAS) (http://www. affymetrix.com/estore/partners_programs/programs/developer/TilingArrayTools/index.affx). The method was based on the generation of a transcription map constructed by collecting neighbor expressed probes, i.e., probes with a smoothed intensity above a given threshold. TAS extended the 
method of Kampa et al. by estimating the significance of differential expression using a Wilcoxon signed-rank test within local windows of a given width, centered on each probe. More recently, the Model-based Analysis of Tiling-arrays (MAT) was introduced by Johnson et al. (Johnson et al., 2006). MAT standardized the probe signal value through a model, eliminating the need for sample normalization. MAT used an innovative function specifically designed to score regions of chromatin immunoprecipitation (ChIP) enrichment, which allowed robust $p$-value and false discovery rate calculations. However, both methods were not able to detect short transcripts. A solution to the segmentation problem was also proposed by Huber et al. (Huber et al., 2006) they used a change point detection algorithm, based on a dynamic programming approach, that determined the global maximum of the log-likelihood of a piecewise constant model. This model provides good performances, but it doesn't take the probes position into account, making data analysis less accurate in partially covered genomes. Finally, Thomassen et al. (Thomassen et al., 2009) described a new approach to address the problem of segmentation using a sliding and expanding window running along the genomic coordinate. However, the size of the windows employed by Thomassen et al. could assume only three values making the approach narrowly applicable.

Despite the availability of all these algorithms (Kampa et al., 2004; Huber et al., 2006; Johnson et al., 2006; Thomassen et al., 2009), a comprehensive available pipeline for high-density array data analysis is still lacking, in particular for bacterial transcriptomes for which an increasing amount of genome annotations are becoming more and more accessible (Land et al., 2015; Loman and Pallen, 2015). Henceforth, at our knowledge, no existing tool is able to analyse more than one experiment at the same time especially if also the non-coding portions of the genomes are probed.

To address these needs, we have developed PIPE-chipSAD, a pipeline to conduct high-density array data analysis. The main algorithm is chipSAD (chip Signal Areas Detector), which provides the segmentation of the hybridization signal and defines the boundaries of the detected transcripts. Anno-chipSAD performs an annotation of these regions guiding to the identification of new architectural features as operons, small-RNAs and antisense messenger RNAs. Finally, align-chipSAD identifies the transcriptional units analyzing multiple experiments from different chip layouts at the same time.

Herein we provide explications of the main steps of PIPEchipSAD and details of the algorithms strategies. We also show the application of PIPE-chipSAD on two previously published datasets (Mellin et al., 2010; Fagnocchi et al., 2015) used to study the transcriptome variation of Neisseria meningitidis $\triangle h f q$ mutant strain to show the ability of PIPEchipSAD to handle experiments with different experimental designs.

\footnotetext{
Abbreviations: CPR, correlated probe region, ie clusters of contiguous probes having a correlated signal; SAS, signal areas, ie putative transcribed regions; ORF, open reading frame; UTR, untranslated transcribed region.
}

\section{MATERIALS AND METHODS}

PIPE-chipSAD was designed to investigate two color microarray datasets, but it is suitable to analyse data from different sources (one color microarray or sequencing technologies such as RNAseq) with minimal data elaboration.

The pipeline is composed by five steps, as indicated in the flow chart shown in Figure 1, that correspond to three main programs. This module structure provides more flexibility in the data analysis because the user can access to the different programs independently. The pipeline is freely available at: https:/github.com/silviamicroarray/chipSAD.

\section{Identification of Transcriptional Units}

chipSAD is the first program of the pipeline. The main algorithm defines pairs of contiguous sliding windows along the genomic coordinate $x$. The width of the windows is iteratively increased to include contiguous probes with a consistent signal. Finally, two correlated probe regions (CPRs) of different sizes are positioned at the two sides of each coordinate on the genome (Figure 1A in set 1 (red boxes)).

Then, it follows the identification of positions where the $M$ value signal is subject to an abrupt change of intensity. A generalized $t(x)$ parameter to compare the right and left CPR was defined as in (Tusher et al., 2001) in order to establish the signal "change points." To create a signal area (SAS) a strategy based on the intensity value of contiguous CPRs was employed (Figures 1B,C). In case of tiling probes design, the evaluation of the $t$-test curve is enough to assess the starts and the ends of the SAS, as shown in Figure 1B. On the contrary, in case of non-tiling arrays (i.e., non uniform density of probes) shown in Figure 1C, the analysis of the $t$-test curve is not sufficient to provide good boundaries assessment thus the procedure is enriched with a criterion based on the $M$ value comparison and a signal smoothing calculation based on the pseudomedian or Hodges-Lehmann estimator (Royce et al., 2007). Briefly, the averaged intensity of two regions, each limited by two consecutive change points, was compared: if their pseudomedian intensity was greater than the absolute value of the difference between the pseudomedian intensities of the two regions, they were joined and the comparison would extend to the next region, otherwise two different SAS were created. The result of this step is the list of SAS to be interpreted as putative transcripts.

\section{Comparative Analysis of Multiple Experiments}

In case of multiple experiments, align-chipSAD can be run. In order to avoid that noisy areas may influence the results of this procedure, only the SAS with a minimum value of the M pseudomedian $(\overline{\bar{M}})$ might be selected before running alignchipSAD. Then, graphs based on the overlap of the SAS are built as indicated in Figure 1D by the orange links. For each connected graph, the consensus boundaries are calculated considering a weighted average of the boundaries of the SAS belonging to the graph. In detail, the weighted position $\langle x\rangle$ (start or end) of the 


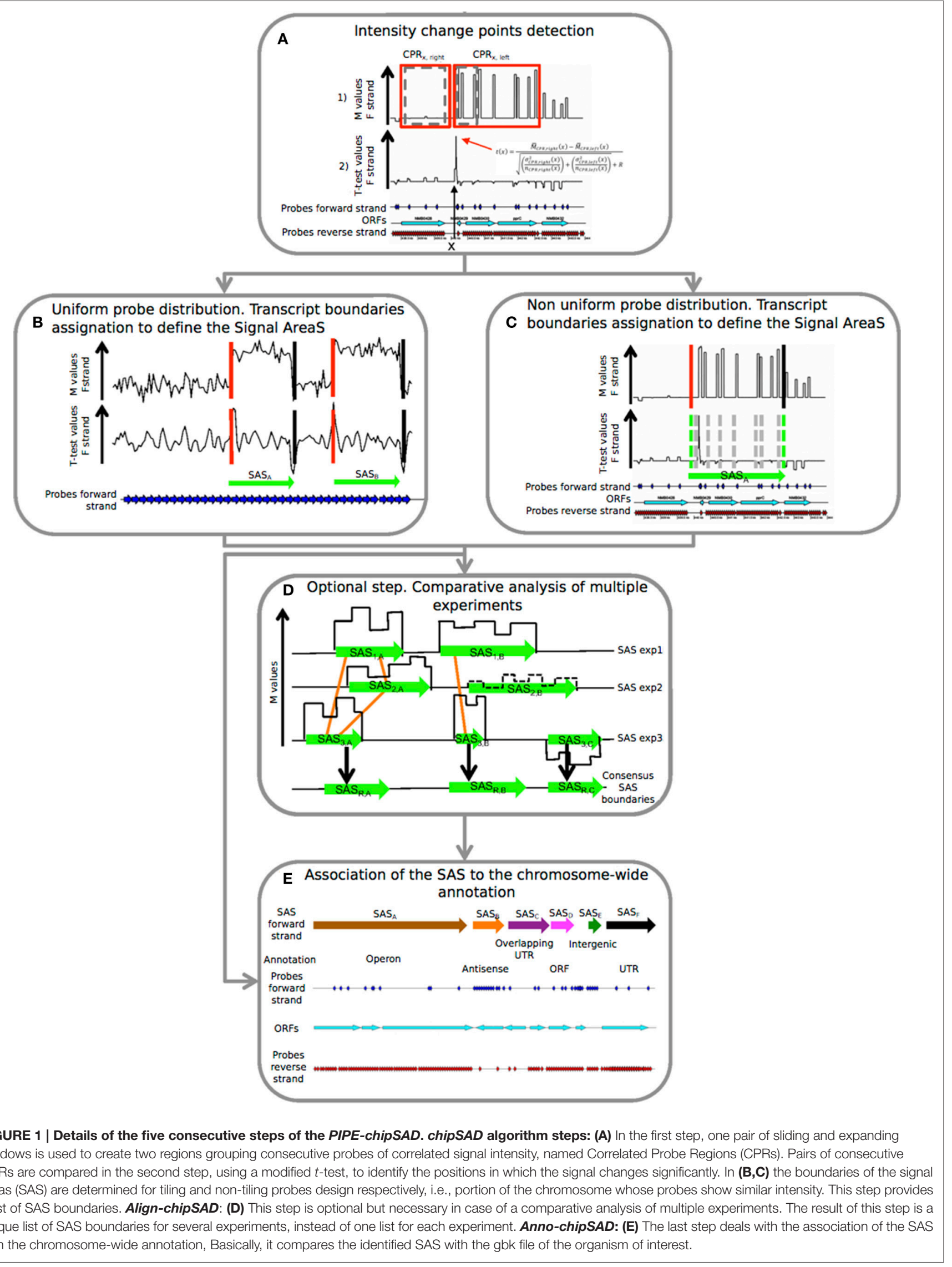


consensus SAS is:

$$
\langle x\rangle=\frac{\sum_{i} p_{i} x_{i}}{\sum_{i} p_{i}} \text { where } p_{i}=\frac{\overline{\bar{M}}_{i}}{\sum_{i} \overline{\bar{M}}_{i}}
$$

with $i=1,2 \ldots, N$ enumerates each SAS belonging to the graph.

Thus, for each connected graph, a consensus SAS is determined, and the conclusion of this step is a unique list of consensus SAS.

\section{Putative Transcripts Annotation}

The final step of the PIPE-chipSAD is the comparison of the detected SAS with the genome annotation and the classification of putative transcripts when they represent single open reading frames (ORFs), entire operons (polycistronic transcripts), antisense RNAs, untranslated transcribed regions (UTRs) and intergenic RNAs (Figure 1E). This procedure consists in the comparison of the identified SAS with the gbk file of the organism of interest and is implemented by anno-chipSAD; comparing the identified SAS with the gbk file of the organism of interest. The first step of the classification was the selection of SAS overlapping annotated ORFs: if the SAS overlapped a minimum of $30 \%$ of a single ORF length in the same strand then it was classified as ORF (magenta arrow in Figure 1E), otherwise as antisense (orange arrow in Figure 1E). If the SAS overlapped two or more co-oriented ORFs in the sense strand (with a minimum of $30 \%$ of each ORF length), then it was classified as operon (brown arrow in Figure 1E). If the SAS overlapped two differently oriented ORFs and the intergenic region between the two ORFs was less than $30 \mathrm{bp}$, then it was classified as overlapping UTR (purple arrow in Figure 1E). If the SAS did not overlap an ORF in both strands and its length was less than 800 bases, it was classified as intergenic RNA (dark green arrow in Figure 1E). Finally, if the SAS overlapped both an ORF and the flanking intergenic region, at $5^{\prime}$ or $3^{\prime}$ ends, then it was classified as UTR ( $5^{\prime}$ or $3^{\prime}$ respectively) only if the gap between the ORF and the intergenic region is less than 30 bases (black arrow in Figure 1E).

\section{Datasets}

We analyzed two previously published datasets used to study the transcriptome variation of $N$. meningitidis $\Delta h f q$ mutant strain with respect to wild type. The transcriptome data of the $N$. meningitidis MC58 $h f q$ null mutant $(\Delta h f q)$ strain grown in GC medium described by Fagnocchi et al. (Fagnocchi et al., 2015) and the dataset presented by Mellin et al. (Mellin et al., 2010), which examined the $N$. meningitidis MC58 hfq null mutant and the relative complemented strain, grown in iron depleted $\left(100 \mu \mathrm{g}\right.$ desferal $\left.\mathrm{ml}^{-1}\right)$ or replete conditions $(100 \mu \mathrm{g}$ ferric nitrate $\mathrm{ml}^{-1}$ ), were used in this study, to test the capability of chipSAD to handle data from different experimental designs. The microarrays analyzed are one or two-color hybridizations. In case of two-color experiments (Fagnocchi et al., 2015) a competitive hybridization between the null mutant and the wild type strains growth under the same in vitro growth condition was performed. In case of one-color experiments (Mellin et al., 2010) an in silico comparison to wild type strains growth was composed during global normalization. Three biological replicates of each experimental condition were available in each dataset. In order to make the data comparable, we merged the replicas of the same condition by averaging the $\mathrm{M}$ value of each probe after a global normalization of signals by using limma R package (Smyth and Speed, 2003).

\section{Parameter Estimation}

Automatic parameter estimation was done by the analysis of the distribution of probe $M$ values. The determination of a suitable initial window size $w$ was constrained by the spacing of the probes along the chromosome. Thus, the window size $w$ was estimated calculating the average distance between consecutive probes. The parameter $m(w)$ was estimated by calculating the minimum number of probes found in a window with size $w$.

\section{RESULTS AND DISCUSSION}

\section{Comparative Analysis of the Transcriptome of $\boldsymbol{N}$. meningitidis $\Delta h f q$ Mutant in Different Growth Conditions}

We analyzed two previously published datasets (Mellin et al., 2010; Fagnocchi et al., 2015) used to study the transcriptome variation of Neisseria meningitidis $\Delta h f q$ mutant strain to test the capabilities of chipSAD to manage different chip designs and hybridizations in a single analysis (Figure 2).

In Table $1 \mathrm{SM}$ is reported the number of differentially expressed transcripts for each genomic category found by PIPEchipSAD after the alignment of the two datasets. The application of PIPE-chipSAD allowed the detection of differentially expressed transcripts, especially for transcripts located in intergenic regions, in the five samples without affecting the results obtained running chipSAD on a single sample. In order to see how the alignment procedure, implemented in align-chip $S A D$, affects the SAS identified by chipSAD, we independently ran the chipSAD algorithm only on the $\Delta h f q$ mutant experiment of Fagnocchi's dataset and on the all experiments together and we compared the results. Among the 114 regions classified as single ORFs detected in the single run, 104 are still present after the alignment: 84 of those maintain the same classification, while 20 regions are rearranged in different kind of transcripts mainly as mRNA with UTRs or operons. Ten transcripts are no longer detected as differentially expressed after the application of align-chipSAD. A very similar scenario is observed with operons and mRNAs with UTRs. The 93 mRNAs with UTRs identified in the analysis of a single experiment overlap 115 transcripts (some mRNAs with UTRs comprise two genes), 74 of them are still classified as mRNAs with UTRs after the alignment procedure, while 33 transcripts are classified in a different class, more often in single ORFs than in operons. Finally, 100 out of 123 transcripts classified as operons belong to the same classification with the single run and upon align-chipSAD application. The intergenic regions are the most affected by the alignment procedure, because these regions usually are short and noisy. One hundred twentysix intergenic regions out of 177 survive to the alignment of which 32 are joined in longer transcripts such as mRNA with UTR or operon. Twenty-seven intergenic regions are no longer present mainly because they had $\mathrm{M}$ values close to the threshold. 


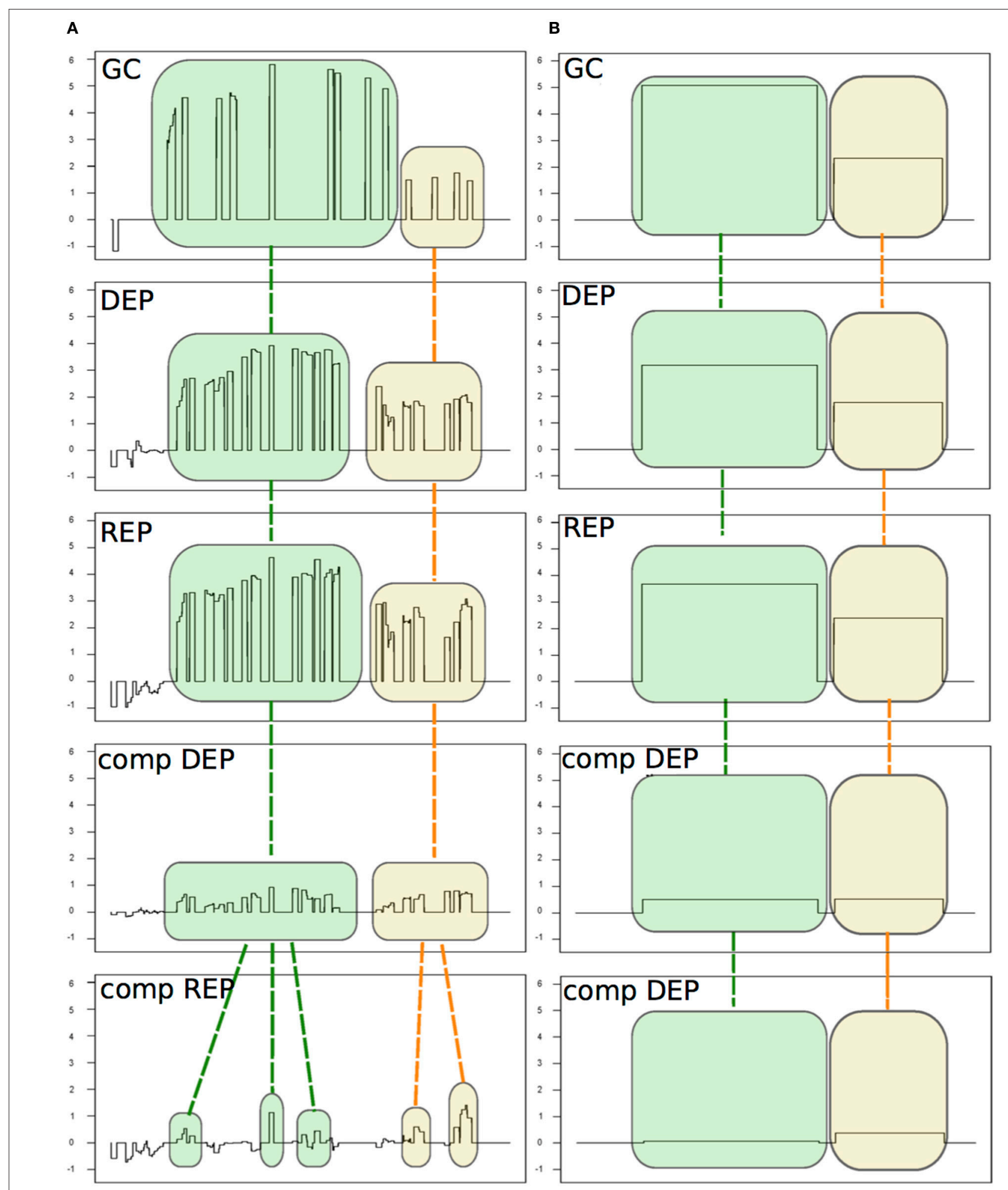

FIGURE 2 | An example of the alignment procedure of the detected signal areas (SAS). The five experiments are: $\Delta h f q$ mutant growth in GC medium (GC, from Fagnocchi et al., 2015) and four experiments from Mellin et al. (2010): the $\Delta$ hfq mutant and the complemented mutant (comp) grown under iron depleted (DEP) or replete (REP) conditions. (A) The SAS identified by chipSAD for the five experiments before the run of the alignment procedure. (B) The aligned SAS. 
Therefore, the main effect of the alignment procedure is the rearrangement of the regions in different classes respect to the classification obtained applying chipSAD on a single experiment. Anyway, after the alignment, only a small percentage of the transcripts have a class change, meaning that the boundaries of the regions detected by chipSAD, even considering only one experiment, are highly conserved. Moreover, only few transcripts are no longer detected after the alignment and most of them had very low $M$ value, meaning that the align-chipSAD can improve the detection of significant regions removing noisy regions.

In order to compare our results with the panel of 132 genes differentially expressed already published in Mellin et al. (Mellin et al., 2010), we first selected the transcripts specifically differentially expressed in the $\Delta h f q$ mutants with respect to both the wild type and also the complemented strain. Thus, we set up a threshold on the $M$ value, selecting only those transcripts with a minimal expression fold change of 2 between the $\Delta h f q$ or the complemented vs wild type strain, in each growth condition. This selection criterion led to the identification of 90 transcripts, including 35 ORFs, 28 operons, 10 mRNA with UTRs, 17 intergenic regions (Figure 3), that are the most reliably and consistently Hfq-modulated and iron-dependent transcripts. Noteworthy, we found that 42 genes were identified by both approaches, however PIPE-chipSAD was able to determine whether these transcripts were organized in more complex structures such as operons or mRNA with UTRs. Furthermore, we individuated 31 previously unidentified Hfq-modulated and iron-dependent transcripts. Finally, our approach found 17 new intergenic regions to be specifically deregulated under iron starvation/abundance and in absence of $\mathrm{Hfq}$, providing also the specific boundaries of the putative transcripts. Overall our method allowed a better understanding of the Hfq targetome. Finally, the results obtained by the application of align-chipSAD made the comparison of transcripts identified across datasets much easier, allowing a compact view such as a heat map (Figure 3).

We have presented PIPE-chipSAD, a specifically designed pipeline for bacterial transcriptomic analysis of high-density array data. The workflow is articulated in three main programs: chipSAD, align-chipSAD and anno-chipSAD. We previously used chipSAD, to analyse the transcriptome variation of $N$. meningitidis in a time course adaptation to human blood (Del Tordello et al., 2012) and in response to physiologically relevant growth conditions (Fagnocchi et al., 2015). These two successfully applications showed the widely applicability of this tool and the reliability of the achieved results. Here, the method was improved to achieve better performances in the segmentation of the intensity signal for both uniform and not uniform probe array designs (i.e., tiling and non-tiling microarray design). Moreover, we have showed that align-chipSAD was able to manage several experiments, with different experimental design, analyzing them simultaneously. Furthermore, we have demonstrated that PIPEchipSAD allows tracing and studying the transcriptional profile of both coding and non-coding portions of the chromosome in a single framework. Overall, bearing the versatile characteristics of PIPE-chipSAD, we believe that it will be of wide applicability

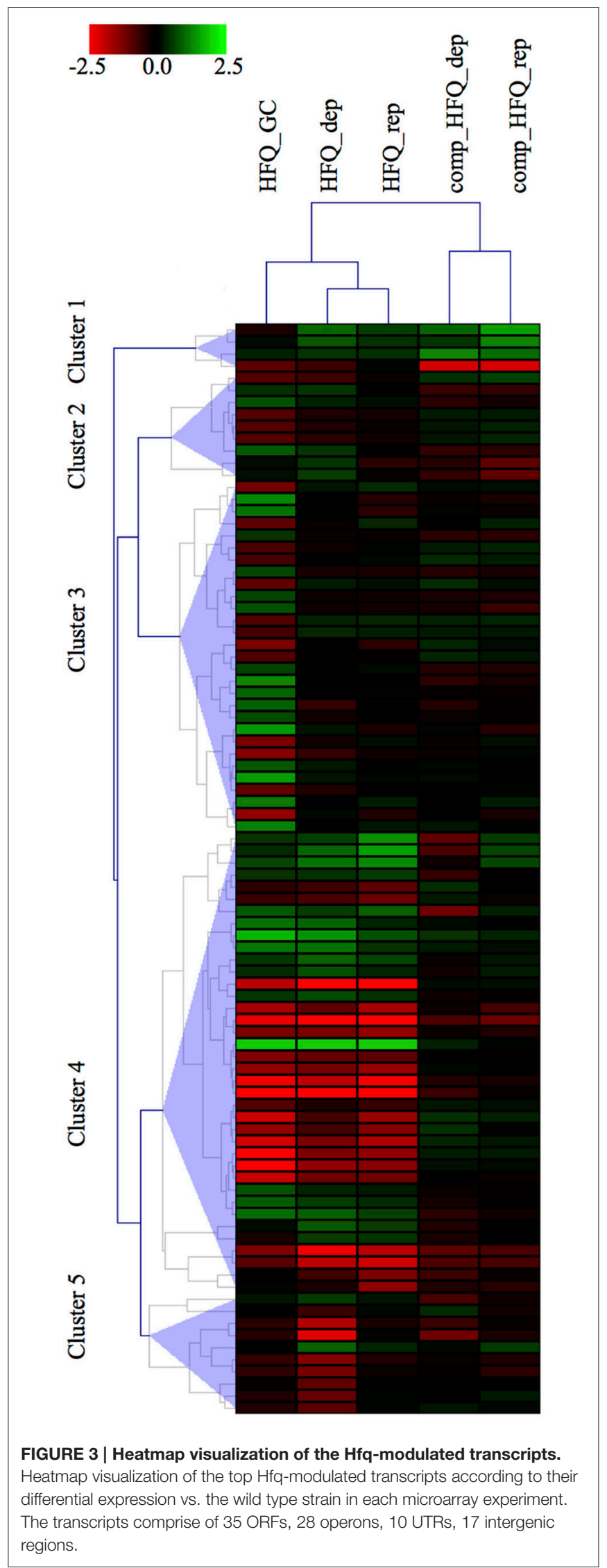


and it might be easily applied to analyse data from other highthroughput technologies such as RNA-seq.

\section{AUTHOR CONTRIBUTIONS}

SB and AM designed and conceived the study. SB developed the software and analyzed the data. SB, ED, LF, CD, and $\mathrm{AM}$ contributed to development of the analysis tool and to results interpretation. SB and AM wrote the manuscript. All authors contributed, provided comments and approved the final manuscript.

\section{FUNDING}

SB was recipient of a Novartis Vaccines fellowship from the Ph.D. program of the University of Siena. LF and ED were recipient

\section{REFERENCES}

Aparicio, O., Geisberg, J. V., and Struhl, K. (2004). Chromatin immunoprecipitation for determining the association of proteins with specific genomic sequences in vivo. Curr. Protoc. Cell Biol. Chapter 17, Unit 17. 7. doi: $10.1002 / 0471143030 . c b 1707 \mathrm{~s} 23$

Bertone, P., Stolc, V., Royce, T. E., Rozowsky, J. S., Urban, A. E., Zhu, X., et al. (2004). Global identification of human transcribed sequences with genome tiling arrays. Science 306, 2242-2246. doi: 10.1126/science.1103388

Crawford, G. E., Davis, S., Scacheri, P. C., Renaud, G., Halawi, M. J., Erdos, M. R., et al. (2006). DNase-chip: a high-resolution method to identify DNase I hypersensitive sites using tiled microarrays. Nat Methods 3, 503-509. doi: $10.1038 /$ nmeth 888

Del Tordello, E., Bottini, S., Muzzi, A., and Serruto, D. (2012). Analysis of the regulated transcriptome of Neisseria meningitidis in human blood using a tiling array. J. Bacteriol. 194, 6217-6232. doi: 10.1128/JB.01055-12

Fagnocchi, L., Bottini, S., Golfieri, G., Fantappiè, L., Ferlicca, F., Antunes, A., et al. (2015). Global transcriptome analysis reveals small RNAs affecting Neisseria meningitidis bacteremia. PLoS ONE 10:e0126325. doi: 10.1371 /journal.pone. 0126325

Heidenblad, M., Lindgren, D., Jonson, T., Liedberg, F., Veerla, S., Chebil, G., et al. (2008). Tiling resolution array CGH and high density expression profiling of urothelial carcinomas delineate genomic amplicons and candidate target genes specific for advanced tumors. BMC Med. Genomics 1:3. doi: 10.1186/1755-8794-1-3

Huber, W., Toedling, J., and Steinmetz, L. M. (2006). Transcript mapping with high-density oligonucleotide tiling arrays. Bioinformatics 22, 1963-1970. doi: 10.1093/bioinformatics/btl289

Johnson, W. E., Li, W., Meyer, C. A., Gottardo, R., Carroll, J. S., Brown, M., et al. (2006). Model-based analysis of tiling-arrays for ChIP-chip. Proc. Natl. Acad. Sci. U.S.A. 103, 12457-12462. doi: 10.1073/pnas.0601180103

Kampa, D., Cheng, J., Kapranov, P., Yamanaka, M., Brubaker, S., Cawley, S., et al. (2004). Novel RNAs identified from an in-depth analysis of the transcriptome of human chromosomes 21 and 22. Genome Res. 14, 331-342. doi: $10.1101 /$ gr.2094104

Land, M., Hauser, L., Jun, S. R., Nookaew, I., Leuze, M. R., Ahn, T. H., et al. (2015). Insights from 20 years of bacterial genome sequencing. Funct. Integr. Genomics 15, 141-161. doi: 10.1007/s10142-015-0433-4

Liu, X. S. (2007). Getting started in tiling microarray analysis. PLoS Comput. Biol. 3:30183. doi: 10.1371/journal.pcbi.0030183 of a Novartis Vaccines fellowship from the Ph.D. program of the University of Bologna. The author AM was employed by the funding organization, Novartis Vaccines now a GSK company.

\section{ACKNOWLEDGMENTS}

The authors would like to thank D. Serruto, I. Delany, and M. De Chiara for helpful discussions and for help in editing the manuscript.

\section{SUPPLEMENTARY MATERIAL}

The Supplementary Material for this article can be found online at: http://journal.frontiersin.org/article/10.3389/fmolb. 2016.00082/full\#supplementary-material

Loman, N. J., and Pallen, M. J. (2015). Twenty years of bacterial genome sequencing. Nat. Rev. 13, 787-794. doi: 10.1038/nrmicro3565

Mellin, J., McClure, R., Lopez, R., Green, D., Reinhard, O. B., and Genco, C. (2010). Role of $\mathrm{Hfq}$ in iron-dependent and -independent gene regulation in Neisseria meningitidis. Microbiology 156, 2316-2326. doi: 10.1099/mic.0.03 9040-0

Royce, T. E., Carriero, N. J., and Gerstein, M. B. (2007). An efficient pseudomedian filter for tiling microrrays. BMC Bioinformatics 8:186. doi: 10.1186/1471-2105-8-186

Selinger, D. W., Cheung, K. J., Mei, R., Johansson, E. M., Richmond, C. S., Blattner, F. R., et al. (2000). RNA expression analysis using a 30 base pair resolution Escherichia coli genome array. Nat. Biotechnol. 18, 1262-1268. doi: $10.1038 / 82367$

Smyth, G. K., and Speed, T. (2003). Normalization of cDNA microarray data Methods 31, 265-273. doi: 10.1016/S1046-2023(03)00155-5

Thomassen, G. O., Rowe, A. D., Lagesen, K., Lindvall, J. M., and Rognes, T. (2009). Custom design and analysis of high-density oligonucleotide bacterial tiling microarrays. PLoS ONE 4:e5943. doi: 10.1371/journal.pone.0005943

Tusher, V. G., Tibshirani, R., and Chu, G. (2001). Significance analysis of microarrays applied to the ionizing radiation response. Proc. Natl. Acad. Sci. U.S.A. 98, 5116-5121. doi: 10.1073/pnas.091062498

Weber, M., Davies, J. J., Wittig, D., Oakeley, E. J., Haase, M., Lam, W. L., et al. (2005). Chromosome-wide and promoter-specific analyses identify sites of differential DNA methylation in normal and transformed human cells. Nat. Genet. 37, 853-862. doi: 10.1038/ng1598

Zhang, Z. H., Jhaveri, D. J., Marshall, V. M., Bauer, D. C., Edson, J., Narayanan, R. K., et al. (2014). A comparative study of techniques for differential expression analysis on RNA-Seq data. PLoS ONE 9:e103207. doi: 10.1101/005611

Conflict of Interest Statement: The authors declare that the research was conducted in the absence of any commercial or financial relationships that could be construed as a potential conflict of interest.

Copyright (c) 2016 Bottini, Del Tordello, Fagnocchi, Donati and Muzzi. This is an open-access article distributed under the terms of the Creative Commons Attribution License (CC BY). The use, distribution or reproduction in other forums is permitted, provided the original author(s) or licensor are credited and that the original publication in this journal is cited, in accordance with accepted academic practice. No use, distribution or reproduction is permitted which does not comply with these terms. 